\title{
Morphometric assessment of reflux oesophagitis in fibreoptic biopsy specimens
}

\author{
LR JARVIS, J DENT, ${ }^{*}$ R WHITEHEAD \\ From the Departments of Pathology and *Gastroenterology, Flinders Medical Centre, Bedford Park, \\ SA 5042, Australia
}

SUMMARY The oesophageal epithelium of patients with reflux oesophagitis has been studied by means of computer aided morphometry. Measurements of histological features were made on biopsies from six cases before and after treatment. The size and elongation of the nuclei and their variation, the number of nuclei per unit length or per unit sectioned area, and the size and number of nucleoli per nucleus were measured for two zones of the epithelium, the base layer and the intermediate layer, which were independent of section orientation. The measurements were analysed using discriminant analysis. Significant discrimination was found between the two groups. The most important parameters were the number of intermediate layer nuclei per sectioned square millimetre, the mean intermediate layer nuclear area, and the number of nuclei per millimetre of base epithelium. These parameters are consistent with increased cell turnover of the non-ulcerated epithelium before treatment.

As part of a pilot study of the effect on reflux oesophagitis of Omeprazole, a potent drug which inhibits gastric secretion, ${ }^{1-3}$ it was necessary to make an accurate assessment of the oesophageal morphology in biopsy tissues. This paper describes a morphometric method which overcomes the problems common to subjective assessment of these small tissue fragments.

\section{Material and methods}

MATERIAL

Six patients with typical reflux oesophagitis participated in the study. At the first endoscopic examination oesophageal ulceration, confirmed by biopsy, was present. Three or four other biopsies were taken in a circumferential fashion from non-ulcerated mucosa between 3 and $5 \mathrm{~cm}$ from the cardiooesophageal junction. Endoscopy was repeated eight weeks after treatment with Omeprazole and in all cases the ulcers had healed. Further biopsies were taken as before. The biopsies were fixed in glutaraldehyde and processed into epoxy resin. Two micron thick sections were cut and stained with haematoxylin and eosin.

Accepted for publication 4 October 1984

\section{DEFINITION OF TISSUE ZONES}

No attempt was made to orientate the small tissue fragments during processing. Consequently, the plane of sectioning was random and did not allow study of orientation dependent features such as increased height of papillae and relative proportions of epithelial zones. It was, however, possible to define tissue zones which were independent of section orientation. These zones were the dividing base layer of the epithelium and the cell layer mid-way between the base and the surface, hereafter referred to as the intermediate layer.

In all cases examined the section orientation was such that some of the papillae were cut in cross section and were recognised as circular or oval profiles. The base layer was defined as that single layer of cells immediately surrounding the core of lamina propria constituting the papillae (Fig. 1). The intermediate layer was defined as the region mid-way between the base and the surface and the correct level was recognised by clear evidence of a core of lamina propria in the cross sectioned papillae (Fig. $2)$. In these zones features which were considered to reflect reaction to cell damage by increased cell turnover were measured.

It was reasoned that increased division in the base layer would result in more nuclei per unit length of sectioned base layer epithelium, elongated nuclei 


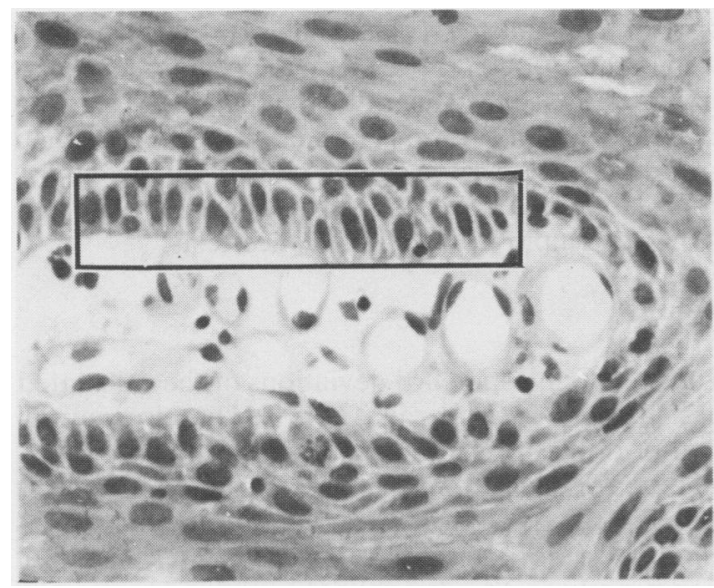

Fig. 1 Section showing the dividing base layer of epithelium. The sampled area used for nuclear measurements is outlined.

due to physical crowding, and a smaller average and more variable nuclear size owing to a higher proportion of dividing (smaller) nuclei. Furthermore, increased cell turnover would lead to more nuclei in the intermediate layer and a smaller average size owing to reduced time for nuclear growth. Nucleolar size and numbers would also be expected to be greater with increased synthetic activity of the nuclei. These changes would be reflected in the mean size and shape of the nuclei and their variation, the mean number of nuclei per unit length or per unit area, and the mean size and number of nucleoli per nucleus.

\section{SAMPLING}

Segments of the base layer epithelium containing at least 20 clear nuclear profiles were chosen (Fig. 1). For the intermediate layer a rectangular sample area was chosen sufficiently distant from the papillar profiles to avoid sampling the more densely stained nuclei of the dividing layers and of sufficient size to contain at least 20 nuclear profiles (Fig. 2). Intraepithelial lymphocytes, easily recognised by their distinctive size and dense staining characteristics, were excluded (Figs. 1 and 2). Nuclei intersecting the field edges were included only for the upper and left hand edges of each field. At least 10 segments of base layer epithelium and 10 areas of the intermediate layer were selected for each case. The sampling numbers were chosen empirically to give a consistent average by the principle of summation averages. ${ }^{4}$

\section{MEASUREMENT METHODS}

Drawings of the features to be measured were made with the aid of a microscope drawing tube attach-

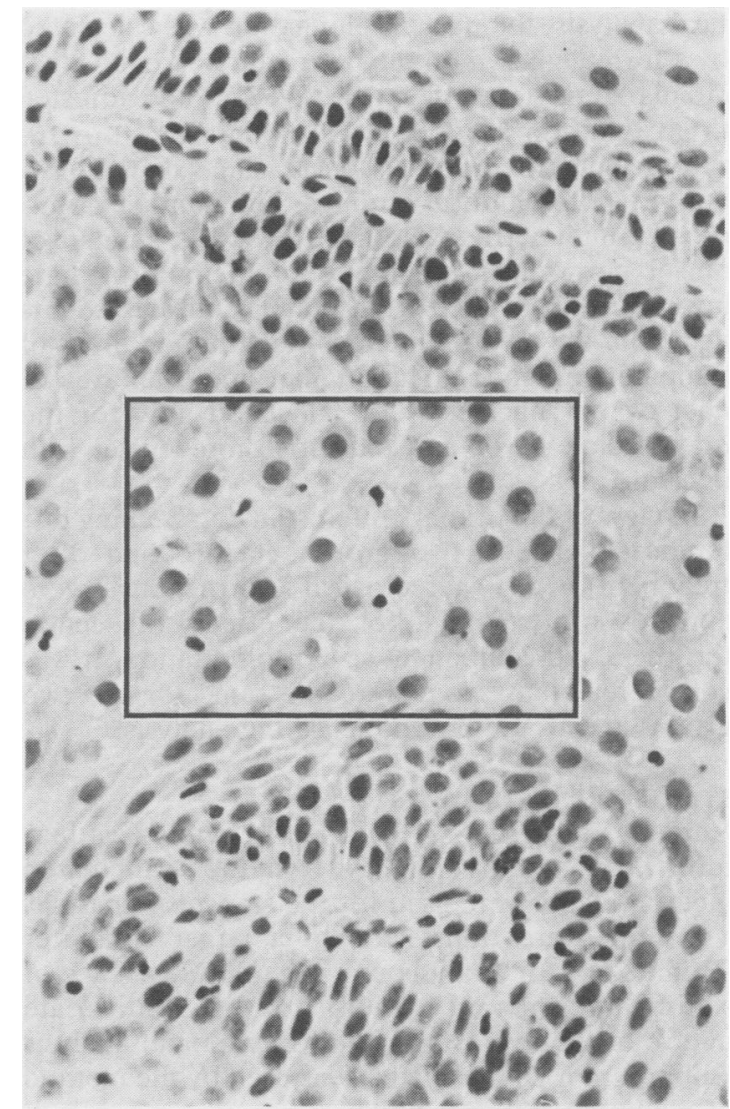

Fig. 2 Section showing the intermediate layer. The sampled area is outlined and illustrates the distinction between the intermediate layer nuclei and the more dense nuclei of the dividing layers. A core of lamina propria visible in the sectioned papillae defines the region of the intermediate layer. The small densely staining lymphocytes are obvious.

ment. A $40 \times$ microscope objective gave an image magnification on the drawing surface of 1190 . The drawings were measured using a digitiser tablet system. The system comprised a microcompter (North Star Advantage), digitiser tablet (Houston Instruments Hipad), graphic printer (Epson FX-100), and measurement software developed in our laboratory.

The nuclear profiles and the length of the mid-line of the epithelium were measured for each base layer segment. The nuclear profiles and the sampled area were measured for each field of the intermediate layer. The basic measurements made on each image feature were simply the area and the perimeter.

CALCULATION OF PARAMETERS

Nuclear area was calculated in micrometres directly using a calibration factor. For convenience in statis- 
tical analysis, the nuclear elongation was calculated by a formula based on the perimeter and equivalent circular area $\left(10-40 \times\right.$ Area/Perim $\left.{ }^{2}\right)$ and adjusted to give an arbitrary scale with limits from 0 to 10 , zero being a true circle and increasing values denoting the more elliptical outlines. The number of nuclei per millimetre of the base layer and number of nuclei per square millimetre of the intermediate layer were calculated from manual nuclear counts and from the measurements of lengths and areas of sampled epithelium. The number and total area of nucleoli per nucleus were calculated only for the intermediate layer and only for those nuclei with nucleoli.

In view of the primary interest in determining differences between two groups (before and after treatment), no stereological corrections were applied-for example, for the effects of section thickness and component size or orientation. It was considered that errors were the same for the two groups and would not influence the analysis.

\section{STATISTICAL ANALYSIS}

Variables representing the selected cytological features calculated for each case from the accumulated measurements were used for the statistical analysis. The data were transferred to a larger computer (Prime 850) for analysis using the SPSS package of statistical programs. ${ }^{5}$ Owing to the multivariate nature of the results, stepwise discriminant analysis (subprogram Discriminant) was used by the method of minimum Wilk's lambda.

Discriminant analysis is a multivariate statistical technique which reveals the effectiveness and relative importance of variables in distinguishing predefined groups of cases. The method may also be used for subsequently classifying unknown cases from classification equations derived from the discriminant functions. The analysis is performed by including variables in a stepwise fashion and calculating at each step the effectiveness of the included variables in discriminating the predefined groups. The analysis is stopped when significant discrimination is achieved. A probability level of $p<$ 0.05 was employed for significance tests.

\section{Results}

The means and standard deviations of the calculated parameters are shown in Table 1. Birect examination of differences in means, however, may be misleading for such a small number of cases if there is high within group variance. Discriminant analysis eliminates those variables which do not contribute significantly to the discrimination between the two groups. Only those variables which are consistently different (low within group variance) are retained.

The analysis reduces the original 13 variables to one discriminant function derived from three variables (Table 2). The relative magnitude of standardised discriminant function coefficient for each variable gives an indication of their relative discriminating power.

The mean number of intermediate layer nuclei per sectioned square millimetre was higher before treatment (965.95) than after treatment (601.97), indicating more cells per unit volume of tissue before treatment. The mean nuclear size in the intermediate layer was slightly smaller before $(27 \cdot 50$ $\left.\mu \mathrm{m}^{2}\right)$ than after treatment $\left(27.99 \mu \mathrm{m}^{2}\right)$. The fact that the discriminant analysis proved this variable to be an important discriminator with a small difference in means indicates consistency between cases. An increased number of nuclei per millimetre of the base layer, suggesting higher division activity, was found before treatment $(95.70)$ compared with after

Table 1 Means of 13 morphometric variables for oesophageal biopsies from patients before and after Omeprazole treatment

\begin{tabular}{|c|c|c|c|c|}
\hline \multirow[t]{2}{*}{ Variable } & \multicolumn{2}{|c|}{ Before $(n=6)$} & \multicolumn{2}{|c|}{ After $(n=6)$} \\
\hline & Mean & $S D$ & Mean & $S D$ \\
\hline $\begin{array}{l}\text { Mean area of base layer nuclei } \\
\text { Mean area of intermediate layer nuclei } \\
\text { CV of area of base layer nuclei } \\
\text { CV of area of intermediate layer nuclei } \\
\text { Mean shape factor of base layer nuclei } \\
\text { Mean shape factor of intermediate layer nuclei } \\
\text { CV of shape factor of base layer nuclei } \\
\text { CV of shape factor of intermediate layer nuclei } \\
\text { Mean no of nucleoli per intermediate nucleus } \\
\text { Mean total area of nucleoli per intermediate nucleus } \\
\text { Percent nuclei with nucleoli in intermediate layer } \\
\text { Nuclei per square millimetre of intermediate layer } \\
\text { Nuclei per millimetre of base layer }\end{array}$ & $\begin{array}{r}19 \cdot 06 \\
27 \cdot 51 \\
37 \cdot 18 \\
37 \cdot 34 \\
2 \cdot 43 \\
1 \cdot 92 \\
42 \cdot 32 \\
54 \cdot 65 \\
1 \cdot 23 \\
1 \cdot 79 \\
10 \cdot 77 \\
965 \cdot 95 \\
95 \cdot 70\end{array}$ & $\begin{array}{r}4 \cdot 56 \\
8 \cdot 22 \\
2 \cdot 66 \\
4 \cdot 59 \\
0 \cdot 42 \\
0 \cdot 26 \\
6 \cdot 63 \\
3 \cdot 70 \\
0 \cdot 70 \\
1 \cdot 21 \\
12 \cdot 57 \\
170 \cdot 80 \\
20 \cdot 12\end{array}$ & $\begin{array}{r}17.66 \\
28.00 \\
42.83 \\
35.36 \\
2.63 \\
2.06 \\
44.77 \\
48.22 \\
0.98 \\
1.46 \\
6.39 \\
601.97 \\
90.46\end{array}$ & $\begin{array}{r}2.59 \\
3.67 \\
6.16 \\
3.56 \\
0.18 \\
0.34 \\
5.98 \\
4.68 \\
0.50 \\
0.82 \\
10.65 \\
72.43 \\
7.67\end{array}$ \\
\hline
\end{tabular}

SD $=$ standard deviation.

$\mathrm{CV}=$ coefficient of variation. 
Table 2 Discriminant function coefficients

\begin{tabular}{lcc}
\hline & Unstandardised & Standardised \\
\hline $\begin{array}{l}\text { Nuclei per square } \\
\text { millimetre of } \\
\text { intermediate layer }\end{array}$ & 0.0101 & 3.035 \\
$\begin{array}{l}\text { Mean area of intermediate } \\
\text { layer nuclei }\end{array}$ & 0.4348 & 2.771 \\
$\begin{array}{l}\text { Nuclei per millimetre } \\
\text { sectioned base layer }\end{array}$ & 0.1100 & 1.676 \\
CONSTANT & -30.2365 & \\
\hline
\end{tabular}

treatment $(90.46)$. These variables are consistent with an increased cell division rate before treatment.

Most importantly, the $F$ ratio of the discriminant analysis, which indicates the significance level for distances between group centroids, showed significant discrimination between the two groups $(\mathrm{F}$ $=13.072, \mathrm{p}=0.0019$ ).

The variables excluded from the analysis were the nucleolar measurements, the elongation shape factor, and the variations of size and shape of the nuclei. The small number of cases and high within group variance of these variables was such that they did not contribute significantly to the discriminant analysis.

The unstandardised discriminant functions may be used to calculate a discriminant score for each case. When plotted, these scores graphically illustrate the discrimination between the two groups (Fig. 3). As a further check on the effectiveness of the discrimination, the original cases were classified using a classification equation derived from the discriminant functions and the predicted and actual groupings compared. This resulted in $100 \%$ agreement, further illustrating the success of the discrimination.

\section{Discussion}

Patients with reflux symptoms who have no evidence of oesophageal erosions and equivocal endoscopic

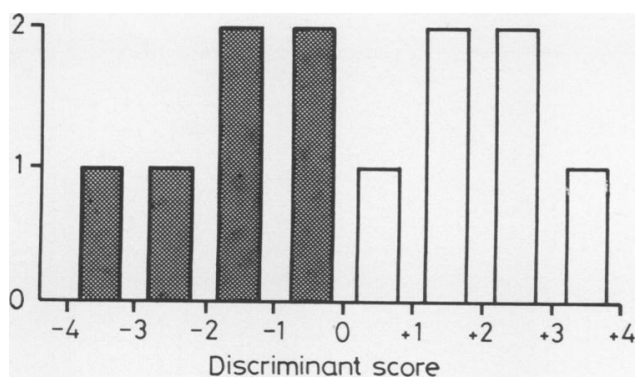

Fig. 3 Histograms of discriminant scores for the cases examined based on the unstandardised discriminant functions and the morphometric variables for each case. The distributions of scores are clearly separated for cases before treatment (solid bars) and after treatment (open bars). appearances ascribable to oesophagitis comprise an appreciable clinical problem in management. Difficulties in assessment of oesophageal biopsies from such patients are also very real because reflux may be regarded as a normal event. Biopsies from the last $2.5 \mathrm{~cm}$ of the oesophagus in normal subjects may show changes ascribed to reflux, ${ }^{6}$ and it is now usual to accept histological evidence of reflux as important only if it is present in the mucosa above this level. Even so, the subjective assessment of the degree of reflux in the biopsy-namely, increased thickness of the basal layer and elongation of the subepithelial papillae ${ }^{7}$-is fraught with difficulty and Adami $e t \mathrm{al}^{8}$ believe that it is of little value. Orientation of minute superficial biopsies is often suboptimal and results in sections cut in a variety of planes through the oesophageal epithelium. Even in correctly orientated sections definition of the upper extent of the basal layer is arbitrary. Although periodic acid Schiff preparations are often used to define the basal layer by its absence of positively stained glycogen, the distinction between the negative and positive zone is never clear cut. Rather there is a gradual transition from positive to negative. One could advocate that the upper limit be taken as the region where the distance between neighbouring nuclei equals the average diameter of the nuclei, but this again is arbitrary and does not allow for real accuracy since the zone with these characteristics is relatively wide. In view of these problems, in this pilot study to assess the effect of Omeprazole, we assessed the lower oesophageal epithelium by morphometric methods. We were able to identify the tissue zone between the basal layer and the surface as well as the single dividing base layer in randomly orientated sections of small biopsy fragments. From these zones we measured 13 different nuclear parameters in biopsy tissues from six patients. Discriminant analysis showed that three of these measurements were important in indicating clear differences between the before and after treatment biopsies. They indicated an increased rate of cell division in response to gastric acid reflux which was significantly different from that when the gastric secretion of acid was inhibited.

Knuff $e t a l^{9}$ have recently shown that in routine punch biopsies of patients with long histories of oesophageal reflux, $59 \%$ were uninterpretable when endoscopic evidence of oesophagitis was minimal. Although in some cases studied here the presence of ulcer debris in the histological sections allowed a firm diagnosis of oesophagitis to be made, the fragments were often too small and unsuitably orientated to allow assessment of accepted indicators of oesophagitis such as increased basal layer thickness or height of papillae, and reliable interpretation was 
not possible by ordinary methods. The importance of the morphometric technique is therefore clear. It shows that there are measurable changes in the non-ulcerated epithelium when reflux of acid is occurring which form an objective basis for the accurate diagnosis of non-ulcerative reflux oesophagitis. This is mandatory if the condition is to be managed by potent drugs.

\section{References}

' Olbe L, Haglund U, Leth R, et al. Effects of substituted benzimidazole (H 149/94) on gastric acid secretion in humans. Gastroenterology 1982;83: 193-8.

${ }^{2}$ Lind T, Cederberg C, Ekenved G, Haglund U, Olbe L. Effect of Omeprazole-a gastric proton pump inhibitor-on pentagastrin stimulated acid secretion in man. Gut 1983;24:270-6.

${ }^{3}$ Konturek SJ, Cieszowski M, Kwiecien N, Konturek J, Tasler J, Bilski J. Effects of Omeprazole, a substituted benzimidazole, on gastrointestinal secretions, serum gastrin, and gastric mucosal blood flow in dogs. Gastroenterology 1984;86:71-7.

${ }^{4}$ Dunnill MS. Quantitative histology. In: Dyke JA, ed. Recent advances in clinical pathology. London: Churchill Livingstone, 1968.

${ }^{5}$ Nie NH, Hull CH, Jenkins JG, Steinbrenner K, Bent DH. Statistical package for the social sciences. 2nd ed. New York: McGraw-Hill Book Company, 1975.

- Weinstein WM, Bogoch ER, Bowes KL. The normal human esophageal mucosa: a histological reappraisal. Gastroenterology $1975 ; 68: 40-4$.

' Ismail-Beigi F, Horton PF, Pope CE. Histological consequences of gastroesophageal reflux in man. Gastroenterology 1970;58: 163-74.

${ }^{8}$ Adami B, Eckardt VF, Paulini K. Sampling error and observer variation in the interpretation of esophageal biopsies. Digestion 1979; 19:404-10.

${ }^{9}$ Knuff TE, Benjamin SB, Worsham F, Hancock JE, Castell DO. Histological evaluation of chronic gastroesophageal reflux. Dig Dis Sci 1984;29:194-201.

Requests for reprints to: Professor R Whitehead, Department of Pathology, Flinders Medical Centre, Bedford Park, South Australia 5042, Australia. 